\title{
UNSTEADY MHD MICROPOLAR FLUID IN A STRETCHING SHEET OVER AN INCLINED PLATE WITH THE EFFECT OF NON-LINEAR THERMAL RADIATION AND SORET-DUFOUR
}

\author{
H. Mondal ${ }^{1}$,S. Mishra ${ }^{2}$, P.K.Kundu ${ }^{3}$, P. Sibanda ${ }^{1}$
}

\begin{abstract}
The effect of unsteady MHD flow of a micropolar fluid over an inclined plate with thermal radiation and non-uniform heat source/sink, non-linear thermal radiation, chemical reaction and convective boundary conditions has been investigated in the present study. A mathematical model is developed to set of Partial differential equations into non-linear coupled ordinary differential equations and then solved numerically by spectral relaxation method (SRM) with finite difference scheme which employs the Gauss-Seidel type of relaxation approach to linearize and decouple the system of differential equations and then Chebyshev pseudospectral method was used to solve the equations. The influence of various physical parameters are depicted graphically and analyzed in details. An excellent agreement of accuracy has found after comparing present work with previously published work.
\end{abstract}

\section{Keywords: Micropolar Fluid, Thermal Radiation, Non-Uniform Heat Source/Sink,} Magnetohydrodynamics, Thermophoresis

\section{INTRODUCTION}

The micro-structured fluids which belongs to a class of fluids with nonsymmetric stress tensor are termed as micropolar fluids. Micropolar fluids having internal micro structures in that coupling between the spin of each particle and these microscope velocity is also considered. This type of structures may be appear in polymer technology. They consists of rigid, randomly oriented or spherical particles with their own spins and micro rotations, suspended in a viscous medium. The study of flow heat mass transfer and angular momentum over a stretching surface is important in many industrial applications such as glass fiber production, the aerodynamic extrusion of plastic sheets, the continuous casting, paper production,glass blowing, metal spinning, drawing plastic films hot rolling and wire drawing. The quality of the final product depends on the rate of heat transfer at the stretching surface. Micropolar fluids are generally non-Newtonian fluids consisting of dump-bell molecules, body fluids colloidal fluids, suspensions fluids, polymers, fluids with additives, and animal blood, etc. There is a considerable increase in the research interest in the flows of micropolar fluids over the past few decades due to the occurrence of these fluids in industrial processes. The theory of micropolar fluids, which display the effect of local rotary inertia and couple stresses, can explain the flow behavior in which the classical Newtonian fluid theory seems to be inadequate. An excellent review of this subject and application of micropolar fluid mechanics has been provided by Pal and Chatterjee. [1,2]. The experimentally that fluids containing minute polymeric additives exhibits a considerable reduction in the skin-friction and hence this concept can be well explained by the theory of micropolar fluids. Thus the study of heat and mass transfer considering micropolar fluid is of special interest because of the cooling of the fibre in the formation process [3]. Abo-Eldahab and El Aziz [4] have considered the flow in a micropolarfluid situations over an isothermal stretching surface embedded in a non-Darcian porous medium. The heat transfer to a micropolar fluid from a non-isothermal stretching sheet with studied by Hady [5]. Ahuja [6] examined that the particle rotation has a important factor in heat transfer enhancement of the fluid. Zaimi and Ishak [7] established the stagnation-point flow and heat transfer of an incompressible micropolar fluid over a nonlinearly stretching/shrinking sheet. El-Aziz [8] analyzed the unsteady mixed convection flow of a viscous incompressible micropolar fluid adjacent to a heated vertical surface with

This paper was recommended for publication in revised form by Regional Editor BekirYilbas

${ }^{1}$ School of Mathematics, Statistics \& Comp. Sci., Uni. of KwaZulu Natal, Private Bag X01, Scottsvile, Pietermaritzburg-3209, South Africa

${ }^{2}$ School of Engineering and Technology, Amity University,New Town, Kolkata-700135

${ }^{3}$ Department of Mathematics, Jadavpur University, Kolkata-700032, India

Orcid id: 0000-0002-9153-300X, 0000-0002-5117-2051, 0000-0002-8487-4893, 0000-0003-2115-4642

${ }^{*}$ E-mail address: hiranmoymondal@yahoo.co.in

Manuscript Received 3 December 2017, Accepted 25 January 2018 
viscous dissipation. Bhargava et al. [9] examined the steady, coupled MHD heat mass transfer of an incompressible micropolar fluid flowing over a nonlinear stretching sheet.MHD heat and mass transfer has gained great interest due to its applications in engineering fields, like extrusion of a polymer sheet, glass fibre and paper production also in many manufacturing processes in modern industry, such as hot rolling, hot extrusion, wire drawing and continuous casting. Thus, research topic of Magnetohydrodynamics (MHD) has been receiving continuous attention all over the world. Pal [10] developed the MHD flow and also heat transfer past a semi-infinite vertical plate embedded in a porous medium. In the field of space technology where operating temperatures is high, radiation effect is quite significant. The knowledge of radiation heat transfer becomes very important, particularly in designing pertinent equipment. Early theoretical investigations in the area of radiative heat transfer were reported by many researchers like Mahapatra et al. [11], Singh [12], Patil et al. [13], Poornima and Reddy [14], Pal and Mondal [15 ], Mostafa [16].

The study of heat source or sink plays an important role in moving fluids. The application of heat generation (source) or absorption (sink) in moving fluids of various physical problem are endothermic chemical reaction or fluids undergoing exothermic. We know that the exact mathematical modelling of the internal heat generation or absorption is little bit problematic, so heat source or sink has been expected to be constant and temperature or space dependent. Chamkha [17] analyzed the MHD flow of a uniformly stretched vertical permeable surface in the presence of heat generation/ absorption and a chemical reaction and unsteady MHD convective heat and mass transfer past a semi-infinite vertical permeable moving plate with heat absorption.

Thermal and solutal convection with chemical reaction has numerous applications in manufacturing processes, for example, in evaporation on the surface of a water body, distribution of temperature, energy distribution in a wet cooling tower, drying, due to freezing the crops are damaged and also in geophysics and volcanic systems $[18,19]$. Ferdows and Qasem [20] investigated the influence of chemical reaction on linearly stretching sheet in a viscous fluid. They reported that, the flow profiles reduces with the increase of Schmidt number and enhances with the increasing the order of chemical reaction. Pal and Mondal [21] studied the influence of thermophoresis and Soret-Dufour on magnetohydrodynamic heat and mass transfer over a nonIsothermal wedge with thermal radiation and Ohmic dissipation.

Motivated by above investigation, authors considered to study theoretically the unsteady thermophoresisSoret-Dufour on MHD flow of a micropolar fluid over an inclined plate with thermal radiation and non-uniform heat source/sink. Present investigated paper were divided into following sections like, mathematical formulations, method of solution, appendix, result and discussion, conclusion, bibliography. In mathematical formulations section, we have discussed problem formulation of governing equations including boundary conditions. In the section of method of solution, we developed the spectral relaxation method (SRM) and its applications to the nonlinear systems partial differential equations. In result and discussion, we tried to find physical insight of various physical parameters through graphical presentation and then draw the conclusion of present investigated problem in conclusion section.

\section{MATHEMATICAL MODEL AND VALIDATION}

Unsteady MHD free convection flow of an incompressible fluid over a vertical stretching surface in a micropolar fluid with thermophoresis particle deposition on considered where a transverse magnetic field $B_{0}$ imposed along $\mathrm{y}$ - axis and induced magnetic field is neglected by assuming a very small magnetic Reynolds number. Also applied magnetic field is considered to be weak so that Hall and ion slip effects may be neglected. The stretching surface has linear velocity $\mathrm{U}_{0}$. Two equal and opposite forces are introduced along the $\mathrm{x}$ - axis so that the sheet is stretched with a speed proportional to the distance from the fixed origin $\mathrm{x}=0$ (see Fig.1). Assume that Dufour effect may be described by a second-order concentration derivative with respect to the transverse coordinate in the energy equation whereas Soret effect is described by the second-order temperature derivative in the mass-diffusion equation. Under the usual boundary approximations, the governing equations are goverened by the following equations (see Aurangzaib [22]): 
Journal of Thermal Engineering, Research Article, Vol. 5, No. 6, Special Issue 10, pp. 205-213,

December, 2019

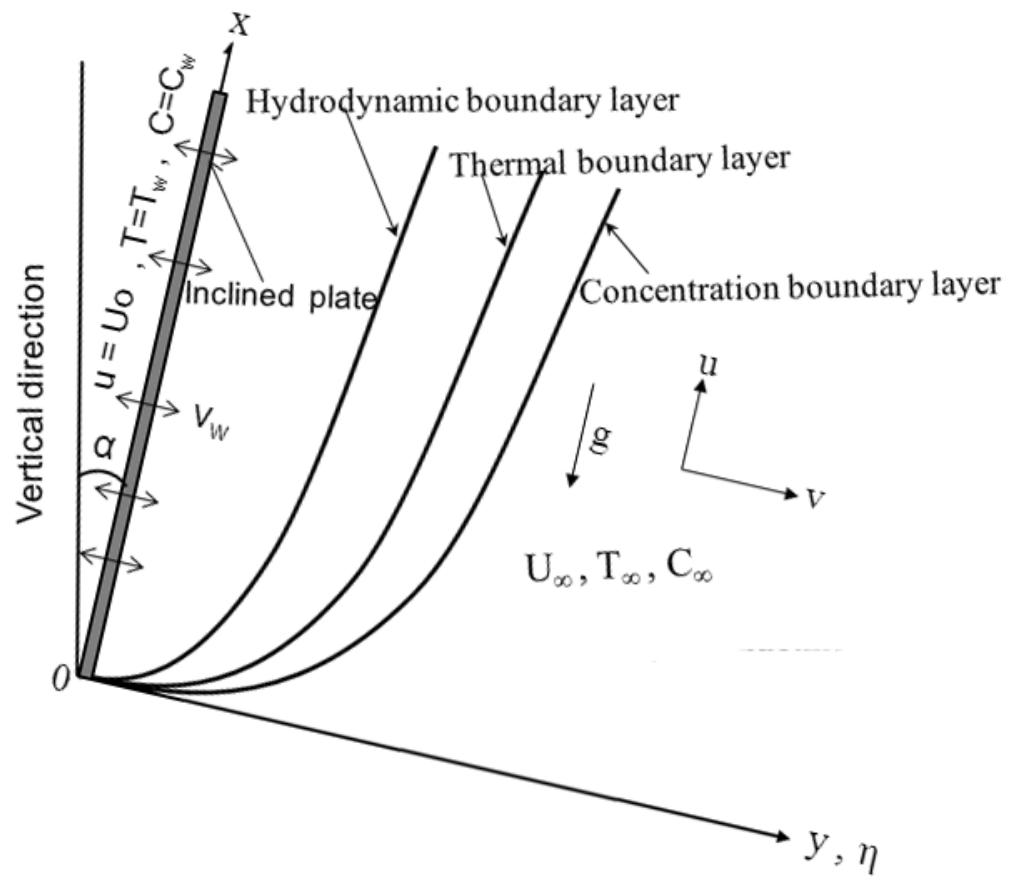

Figure 1. Schematic diagram of the physical model and coordinate system

$$
\begin{gathered}
\frac{\partial u}{\partial x}+\frac{\partial v}{\partial y}=0 \\
\frac{\partial u}{\partial t}+u \frac{\partial u}{\partial x}+v \frac{\partial u}{\partial y}=\left(v+\frac{k_{1}^{*}}{\rho}\right) \frac{\partial^{2} u}{\partial y^{2}}+\frac{k_{1}^{*}}{\rho} \frac{\partial N}{\partial y}-\frac{\sigma B_{0}^{2}}{\rho} u+\left\{g \beta_{T}\left(T-T_{\infty}\right)+g \beta_{c}\left(C-C_{\infty}\right)\right\} \cos \alpha \\
\rho j\left(\frac{\partial N}{\partial t}+u \frac{\partial N}{\partial x}+v \frac{\partial N}{\partial y}\right)=\gamma \frac{\partial^{2} N}{\partial y^{2}}-k_{1}^{*}\left(2 N+\frac{\partial u}{\partial y}\right) \\
\frac{\partial T}{\partial t}+u \frac{\partial T}{\partial x}+v \frac{\partial T}{\partial y}=\frac{\kappa}{\rho C_{p}} \frac{\partial^{2} T}{\partial y^{2}}+\frac{D_{m} k_{T}}{C_{s} C_{p}} \frac{\partial^{2} C}{\partial y^{2}}-\frac{1}{\rho C_{p}} \frac{\partial q_{r}}{\partial y}+\frac{1}{\rho C_{p}} q^{\prime \prime \prime} \\
\frac{\partial C}{\partial t}+u \frac{\partial C}{\partial x}+v \frac{\partial^{2} C}{\partial y}=D_{m} \frac{\partial\left(V_{T} C\right)}{\partial y^{2}}+\frac{D_{m} k_{T}}{\partial y} \frac{\partial^{2} T}{\partial y^{2}}-R^{*}\left(C-C_{\infty}\right)
\end{gathered}
$$

where $\mathrm{u}$ and $\mathrm{v}$ are velocity components along $\mathrm{x}$ - and $\mathrm{y}$ - directions respectively. $\beta_{T}$ and $\beta_{c}$ are coefficients of thermal and concentration expansions. $v$ is the specific heat at constant pressure, $\sigma$ is kinematic viscosity, $\rho$ is electrical conductivity of the fluid, $\mathrm{D}_{\mathrm{m}}$ is the density of the fluid, $\mathrm{N}$ is coefficient of thermal expansion, $\gamma$ is the components of microrotation or angular velocity whose rotation is in the direction of the $\mathrm{x}, \mathrm{y}$ planes and $\mathrm{j}$ is the microinertia per unit mass and $k_{1}^{*}$ is the vortex viscosity.The associated physical boundary conditions are given by 
Journal of Thermal Engineering, Research Article, Vol. 5, No. 6, Special Issue 10, pp. 205-213,

December, 2019

$$
\begin{aligned}
& u=U_{0}=u_{w}=a x, v=0, \quad N=-n \frac{\partial N}{\partial y}, T=T_{w}, C=C_{w} \text { as } y=0 \\
& u \rightarrow 0, N \rightarrow 0, T \rightarrow T_{\infty}, \quad C \rightarrow C_{\infty} \text { as } y \rightarrow \infty
\end{aligned}
$$

The non-uniform heat source/sink q", is modeled as

$$
q^{\prime \prime}=\frac{k u_{w}(x)}{x v}\left[A^{*}\left(T_{w}-T_{\infty}\right) e^{-\eta}+\left(T-T_{\infty}\right) B^{*}\right]
$$

where $\mathrm{A}^{*}$ andB* are the coefficients of space and temperature dependent heat source/sink respectively. Here we make a note that that case $A^{*}>0, B^{*}>0$ corresponds to internal heat generation and $A^{*}<0, B^{*}<0$ corresponds to internal heat absorption. The stream function $\psi$ is defined by $u=\frac{\partial \psi}{\partial y}$ and $v=-\frac{\partial \psi}{\partial x}$, such that the continuity equation (1), is satisfied automatically. The non-dimensional variables are

$$
\begin{aligned}
& \psi=(a v)^{1 / 2} \xi^{1 / 2} x f(\xi, \eta), \eta=\left(\frac{a}{v}\right)^{1 / 2} \xi^{-1 / 2} y, N=\left(\frac{a}{v}\right)^{1 / 2} \xi^{-1 / 2} a x h(\xi, \eta), \xi=1-e^{-\zeta}, \zeta=a t \\
& \theta(\xi, \eta)=\frac{T-T_{\infty}}{T_{w}-T_{\infty}}, \phi(\xi, \eta)=\frac{C-C_{\infty}}{C_{w}-C_{\infty}}, 0 \leq \xi \leq 1
\end{aligned}
$$

The thermophoretic velocity $\mathrm{V}_{\mathrm{T}}$, which appears in Eq. (4), can be written as (Alam et al. [8]):

$$
V_{T}=-\frac{k_{1} v}{T_{r}} \frac{\partial T}{\partial y}
$$

Where $\mathrm{k}_{1}$ is the thermophoresis coefficient, $\mathrm{T}_{\mathrm{r}}$ is the reference temperature.

A thermophoretic parameter $\tau$ can be defined as follows:

$$
\tau=-\frac{k_{1}\left(T_{w}-T_{\infty}\right)}{T_{r e f}}
$$

Employing the Rosseland diffusion approximation the radiation heat flux is given by

$$
q_{r}=-\frac{4 \sigma *}{3 k *} \frac{\partial T^{4}}{\partial y}
$$

where $\sigma^{*}$ is the Stephan Boltzmann constant and $k^{*}$ is the mean absorption coefficient. Let us introduce the wall temperature excessratio parameter as $\theta_{w}=\frac{T_{w}}{T_{\infty}}$. Thus using (8), $\mathrm{T}^{4}$ may be expressed as

$$
T^{4}=T_{\infty}^{4}\left\{1+\left(\theta_{w}-1\right) \theta\right\}^{4}
$$

The nonlinear equations and boundary conditions are reduced to

$$
(1+K) f^{\prime \prime \prime}+K h^{\prime}+\frac{1}{2} \eta(1-\xi) f^{\prime \prime}+\xi\left(f f^{\prime \prime}-f^{\prime 2}-M^{2} f^{\prime}+\left(G r_{t} \theta+G r_{c} \phi\right) \cos \alpha\right)=\xi(1-\xi) \frac{\partial f^{\prime}}{\partial \xi}
$$


Journal of Thermal Engineering, Research Article, Vol. 5, No. 6, Special Issue 10, pp. 205-213,

December, 2019

$$
\begin{aligned}
& \left(1+\frac{K}{2}\right) h^{\prime \prime}+\frac{1}{2}(1-\xi)\left(h+\eta h^{\prime}\right)+\xi\left(f h^{\prime}-f^{\prime} h-K\left(2 h+f^{\prime \prime}\right)\right)=\xi(1-\xi) \frac{\partial h}{\partial \xi} \\
& {\left[\left\{1+N r\left\{1+\left(\theta_{w}-1\right) \theta\right\}^{3}\right\} \theta^{\prime}\right]+\frac{1}{2} \eta(1-\xi) \theta^{\prime}+\operatorname{Pr} \xi\left(f \theta^{\prime}-f^{\prime} \theta\right)+D_{f} \phi^{\prime \prime}+\xi\left(A^{*} e^{-\eta}+B^{*} \theta\right)} \\
& =\xi(1-\xi) \frac{\partial \theta}{\partial \xi} \\
& \quad \frac{1}{S c} \phi^{\prime \prime}+\frac{1}{2} \eta(1-\xi) \phi^{\prime}+\xi\left(f \phi^{\prime}-f^{\prime} \phi\right)-\tau\left(\theta^{\prime} \phi^{\prime}+\phi \theta^{\prime \prime}\right)+\operatorname{Sr} \theta^{\prime \prime}-R_{1} \xi \phi=\xi(1-\xi) \frac{\partial \phi}{\partial \xi}
\end{aligned}
$$

where $K=\frac{k_{1}^{*}}{\mu}$ is the material parameter, $M=B_{0} \sqrt{\sigma / \rho a}$ is the magnetic parameter, $G r_{t}=\frac{g \beta_{T}\left(T_{w}-T_{\infty}\right) x^{3} / v^{2}}{u_{w}^{2} x^{2} / v^{2}}$ is the local Grashof number, $G r_{c}=\frac{g \beta_{c}\left(C_{w}-C_{\infty}\right) x^{3} / v^{2}}{u_{w}^{2} x^{2} / v^{2}}$ is the concentration Grashof number, $D_{f}=D k_{T}\left(T_{w}-T_{\infty}\right) / T_{m} v\left(C_{w}-C_{\infty}\right)$ is the Soret number, $S c=\frac{v}{D}$ is the Schmidt number, $\mathrm{R}_{1}$ is the chemical reaction parameter, $N r=\frac{16 \sigma^{*} T_{\infty}^{3}}{3 k^{*} \kappa}$ is the thermal radiation parameter.

The transformed boundary conditions are

$$
\begin{aligned}
& f(\xi, \eta)=0, f^{\prime}(\xi, 0)=1, h(\xi, 0)=-n f^{\prime \prime}(\xi, 0), \theta(\xi, 0)=1, \phi(\xi, 0)=1, \\
& f^{\prime}(\xi, \eta) \rightarrow 0, h(\xi, \eta) \rightarrow 0, \theta(\xi, \eta) \rightarrow 0, \phi(\xi, \eta) \rightarrow 0, \text { as } \eta \rightarrow \infty .
\end{aligned}
$$

The local skin-friction coefficient in the $\mathrm{x}$ - direction $\mathrm{C}_{\mathrm{fx}}$ the local Nusselt number $\mathrm{Nu}_{\mathrm{x}}$ and the local Sherwood number $\mathrm{Sh}_{\mathrm{x}}$ are defined as follows:

$$
\begin{gathered}
C_{f x}=\frac{\tau_{w}}{\rho u_{w}^{2}}, \tau_{w}=\left[\left(\mu+k_{1}^{*}\right) \frac{\partial u}{\partial y}+k_{1}^{*} N\right]_{y=0} \text { then } C_{f x} \operatorname{Re}_{x}^{1 / 2}=\xi^{-1 / 2}[1+(1-n) K] f^{\prime \prime}(\xi, 0) \\
q_{w}=-\kappa\left(\frac{\partial T}{\partial y}\right)_{y=0}, N u_{x}=\frac{q_{w}}{\left(T_{w}-T_{\infty}\right)}\left(\frac{x}{\kappa}\right) \text { then } N u_{x} \operatorname{Re}_{x}^{-1 / 2}=-\xi^{-1 / 2} \theta^{\prime}(\xi, 0) \\
m_{w}=\rho D\left(\frac{\partial C}{\partial y}\right)_{y=0}, S h_{x}=\frac{m_{w}}{\left(C_{w}-C_{\infty}\right)}\left(\frac{x}{\rho D}\right) \text { then } S h_{x} \operatorname{Re}_{x}^{-1 / 2}=-\xi^{-1 / 2} \phi^{\prime}(\xi, 0)
\end{gathered}
$$

where $\operatorname{Re}_{x}=\frac{u_{w}(x) x}{v}$ is the local Reynolds number based on the surface velocity. 


\section{RESULTS AND DISCUSSION}

In the present study we established unsteady MHD flow of a Micropolar fluid over an inclined plate in the presence of thermal radiation and non-uniform heat source/sink are reported which are then solved by developing the spectral relaxation method (SRM) and its application to the nonlinear systems partial differential equations (13) - (16) with the boundary conditions (17). The results are presented graphically in Figs. 2-7 and conclusions are drawn to analyze the effects of various physical quantities of interest on the velocity, microrotation, temperature and concentration distribution that have significant effects.

Figs. 2-3 shows the effect of material parameter $\mathrm{K}$ on the velocity, and microrotation profiles. We see that velocity profiles increases with the increase of material parameter K. Fig. 3 shows the effect of material parameter $\mathrm{K}$ on the microrotation profiles. From this figure we see that microrotation increases very rapidly with the increase of the material parameter K. It is also understood that as the material parameter increases the rotation of the micropolar constituents gets induced in most part of the boundary layer except very close to the wall where kinematic viscosity dominates the flow.

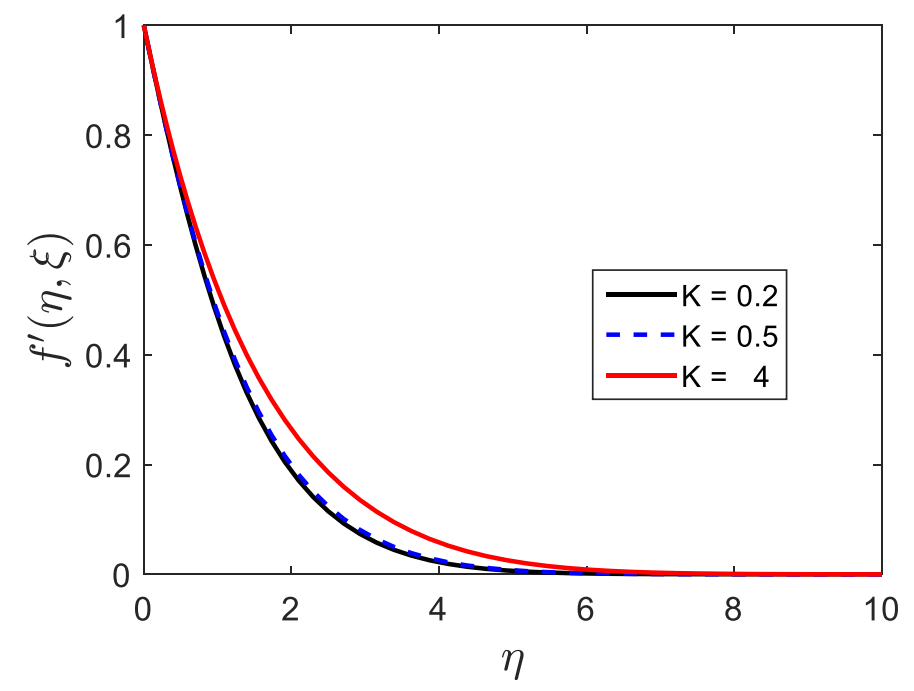

Figure 2. Effect of material parameter $K$ on velocity profile for different values of $\mathrm{n}=0.5, \mathrm{~A}^{*}, \mathrm{~B}^{*}=0.1$, $\alpha=30, \mathrm{Gr}_{\mathrm{t}}=0.1, \mathrm{Gr}_{\mathrm{c}}=0.2, \mathrm{Sc}=2, \operatorname{Pr}=0.7, \mathrm{Nr}=0.3, \mathrm{D}_{\mathrm{f}}=0.2, \mathrm{Sr}=0.4, \mathrm{M}=0.2, \mathrm{R}_{1}=0.5$

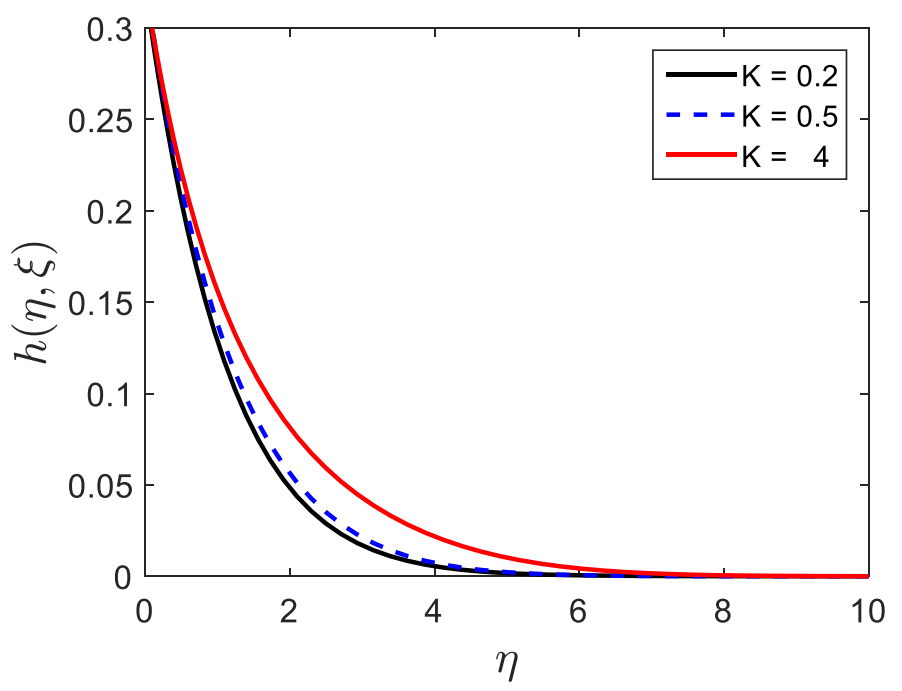

Figure 3. Effect of material parameter $K$ on velocity profile for different values of $\mathrm{n}=0.5, \mathrm{~A}^{*}, \mathrm{~B}^{*}=0.1$,

$$
\alpha=30, \mathrm{Gr}_{\mathrm{t}}=0.1, \mathrm{Gr}_{\mathrm{c}}=0.2, \mathrm{Sc}=2, \operatorname{Pr}=0.7, \mathrm{Nr}=0.3, \mathrm{D}_{\mathrm{f}}=0.2, \mathrm{Sr}=0.4, \mathrm{R}_{\mathrm{l}}=0.5
$$




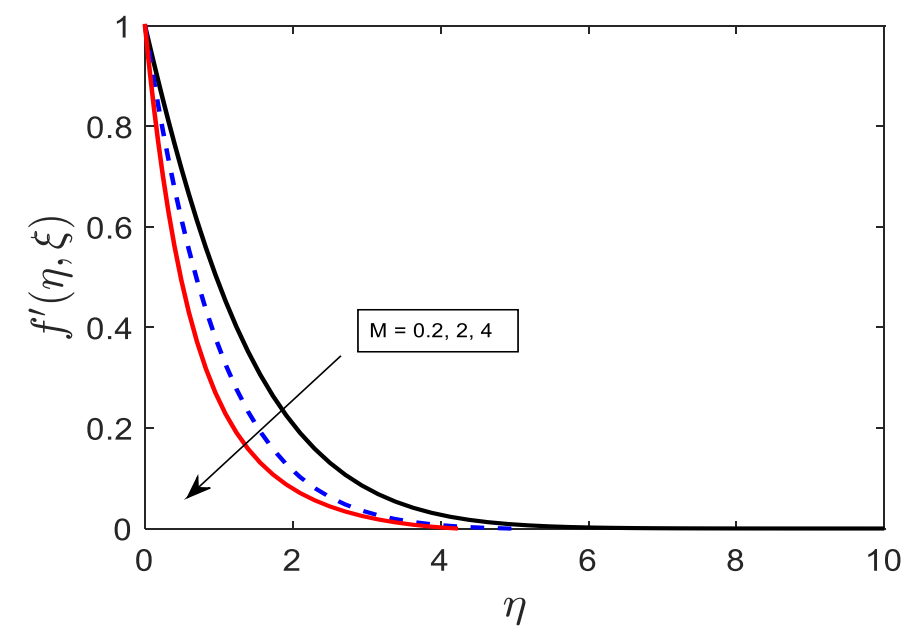

Figure 4.Effect of magnetic parameter $M$ on velocity profile for different values of $\mathrm{n}=0.5, \mathrm{~A}^{*}, \mathrm{~B}^{*}=0.1$,

$$
\alpha=30, \mathrm{Gr}_{\mathrm{t}}=0.1, \mathrm{Gr}_{\mathrm{c}}=0.2, \mathrm{Sc}=2, \operatorname{Pr}=0.7, \mathrm{Nr}=0.3, \mathrm{D}_{\mathrm{f}}=0.2, \mathrm{Sr}=0.4, \mathrm{R}_{\mathrm{l}}=0.5
$$

Figs. 4-5 respectively displays the effect of magnetic field on velocity and microrotation profiles of the flow. It is evident that an increase in the magnetic field parameter $\mathrm{M}$ depreciates the velocity profiles of the flow due to the fact that strengthening the magnetic field causes to enhance the opposite force to the flow is called Lorentz force. Temperature increases due to increase in magnetic field parameter but reverse trend can be observed for microrotation profiles.

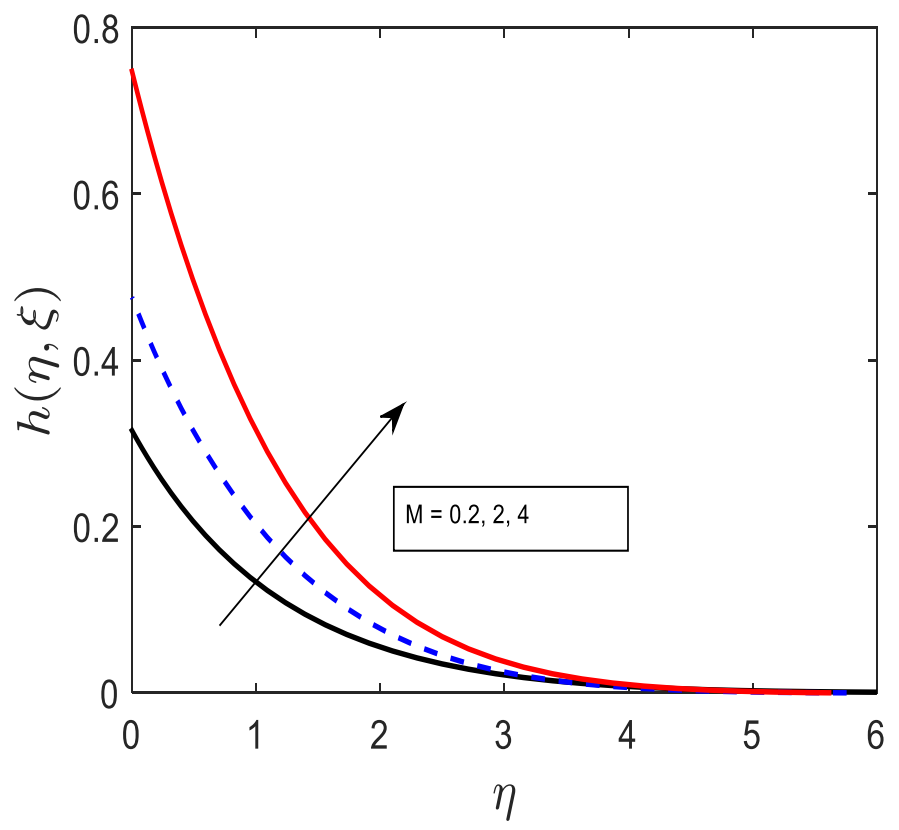

Figure 5. Effect of magnetic parameter $M$ on microrotation profile for different values of $n=0.5, A^{*}, B^{*}=0.1$,

$$
\alpha=30, \mathrm{Gr}_{\mathrm{t}}=0.1, \mathrm{Gr}_{\mathrm{c}}=0.2, \mathrm{Sc}=2, \operatorname{Pr}=0.7, \mathrm{Nr}=0.3, \mathrm{D}_{\mathrm{f}}=0.2, \mathrm{Sr}=0.4, \mathrm{R}_{\mathrm{l}}=0.5
$$

Fig. 6 displays the influence of chemical reaction parameter $R_{1}$ on the concentration profiles. It is seen from this figure that thechemical reaction decelerates the concentration of the fluid.The chemicalreaction rate parameter shows a sharp decrease in the concentrationof the diffusing species. This indicates the fact that theconcentration of the diffusing species reduce during fast reaction. 


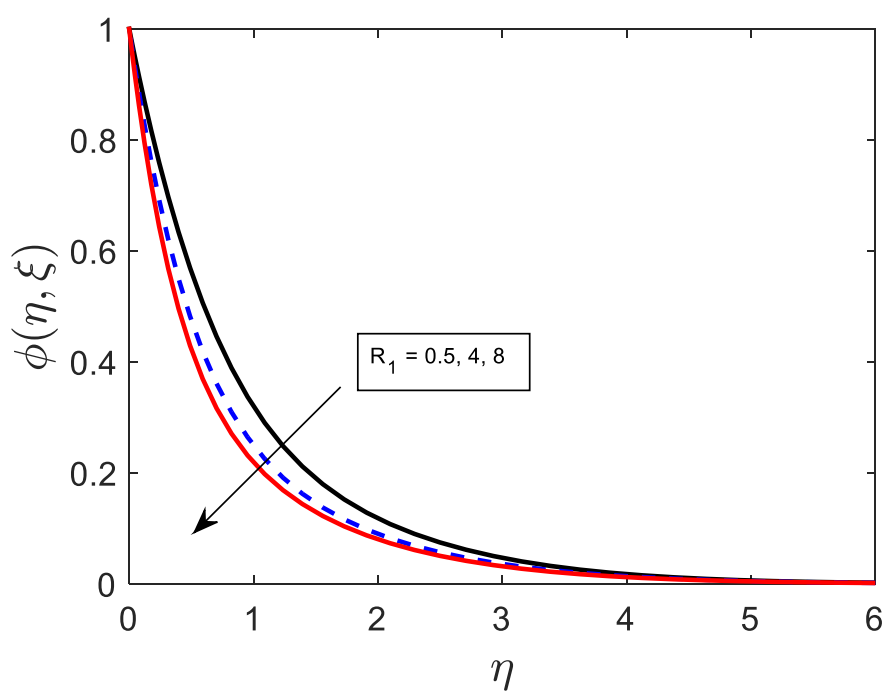

Figure 6. Effects of chemical reaction parameter $R_{1}$ on concentration profile for different values of $n=0.5, A^{*}$,

$$
\mathrm{B}^{*}=0.1, \alpha=30, \mathrm{Gr}_{\mathrm{t}}=0.1, \mathrm{Gr}_{\mathrm{c}}=0.2, \mathrm{Sc}=2, \operatorname{Pr}=0.7, \mathrm{Nr}=0.3, \mathrm{D}_{\mathrm{f}}=0.2, \mathrm{Sr}=0.4, \mathrm{M}=0.5
$$

\section{CONCLUSIONS}

A numerical study has been carried out to analyze the unsteady MHD flow of a micropolar fluid over an inclined plate considering the effect of thermal radiation and non-uniform heat source/sink. Here we consider the spectral relaxation method SRM which is an iterative procedure that employs the Gauss-Seidel type of relaxation approach to linearize and decouple the system of differential equations. The linear terms in each equation is evaluated at the current iteration level (denoted by $\mathrm{r}+1$ ) and non-linear terms are assumed to be known from the previous iteration level (denoted by $\mathrm{r}$ ). Comparisons with previously published work on special cases were performed and results were found to be satisfactory. Effects of different thermo- physical parameter were examined in boundary layer.

Following conclusions are drawn from the numerical results:

- With enhancement of material parameter $\mathrm{K}$ is to decrease micro rotational profile.

- Enhancing magnetic field parameter $\mathrm{M}$ tends to decrease the velocity distribution in the momentum boundary layer.

- Increase in the reaction rate parameter R1 retards the concentration distribution in the solutal boundary layer.

\section{Acknowledgement:}

This work was supported by the Claude Leon Foundation Postdoctoral Fellowship, and the University of KwaZulu-Natal, South Africa.

\section{REFERENCES}

[1] Pal, D., Chatterjee, S. (2010). Heat and mass transfer in MHD non-Darcian flow of a micropolar fluid over a stretching sheet embedded in a porous media with non-uniform heat source and thermal radiation. Communications in Nonlinear Science and Numerical Simulation, 15(7), 1843-1857.

[2] Pal D, Chatterjee S, (2012). MHD Non-Darcy Mixed Convection Stagnation-Point Flow of a Micropolar Fluid Towardsa Stretching Sheet with Radiation, ChemEnggComm, 199865889.

[3] Bourne, D. E., Dixon, H. (1971). The cooling of fibres in the formation process. International Journal of Heat and Mass Transfer, 14(9), 1323-1332.

[4] Abo-Eldahab, E. M., El Aziz, M. A. (2005). Flow and heat transfer in a micropolar fluid past a stretching surface embedded in a non-Darcian porous medium with uniform free stream. Applied Mathematics and Computation, 162(2), 881-899.

[5] F.M. Hady, (1997). Heat transfer to a micropolar fluid from a non-isothermal stretching sheet, Int. J. Num. Meth. HeatFluid Flow 6) 6. 
[6] Ahuja, A. S. (1975). Augmentation of heat transport in laminar flow of polystyrene suspensions. I. Experiments and results. Journal of Applied Physics, 46(8), 3408-3416.

[7] Zaimi, K., Ishak, A. (2014). Stagnation-point flow and heat transfer over a nonlinearly stretching/shrinking sheet in a micropolar fluid. In Abstract and Applied Analysis (Vol. 2014). Hindawi.

[8] El-Aziz, M. A. (2013). Mixed convection flow of a micropolar fluid from an unsteady stretching surface with viscous dissipation. Journal of the Egyptian Mathematical Society, 21(3), 385-394.

[9] Bhargava, R., Sharma, S., Takhar, H. S., Bég, O. A., Bhargava, P. (2007). Numerical solutions for micropolar transport phenomena over a nonlinear stretching sheet. Nonlinear Anal. Model. Control, 12(1), 4563.

[10] Zeiser, T., Lammers, P., Klemm, E., Li, Y. W., Bernsdorf, J., Brenner, G. (2001). CFD-calculation of flow, dispersion and reaction in a catalyst filled tube by the lattice Boltzmann method. Chemical Engineering Science, 56(4), 1697-1704.

[11] Mahapatra, T. R., Nandy, S. K., Gupta, A. S. (2012). Oblique stagnation-point flow and heat transfer towards a shrinking sheet with thermal radiation. Meccanica, 47(6), 1325-1335.

[12] Singh, A. K. (2008). Heat source and radiation effects on magneto-convection flow of a viscoelastic fluid past a stretching sheet: Analysis with Kummer's functions. International Communications in Heat and Mass Transfer, 35(5), 637-642.

[13] Patil, P. M., Chamkha, A. J., Roy, S. (2012). Effects of chemical reaction on mixed convection flow of a polar fluid through a porous medium in the presence of internal heat generation. Meccanica, 47(2), 483-499.

[14] Poornima, T., Reddy, N. B. (2013). Radiation effects on MHD free convective boundary layer flow of nanofluids over a nonlinear stretching sheet. Advances in Applied Science Research, 4(2), 190-202.

[15] Pal, D., Mondal, H. (2010). Hydromagnetic non-Darcy flow and heat transfer over a stretching sheet in the presence of thermal radiation and Ohmic dissipation. Communications in Nonlinear Science and Numerical Simulation, 15(5), 1197-1209.

[16] Mahmoud, M. A. (2009). Thermal radiation effect on unsteady MHD free convection flow past a vertical plate with temperature-dependent viscosity. The Canadian journal of chemical engineering, 87(1), 47-52.

[17] Chamkha, A. (2003). MHD flow of a uniformly stretched vertical permeable surface in the presence of heat generation/absorption and a chemical reaction.

[18] Chamkha, A. J. (2004). Unsteady MHD convective heat and mass transfer past a semi-infinite vertical permeable moving plate with heat absorption. International Journal of Engineering Science, 42(2), 217-230.

[19] Geridönmez, B. P. (2018). Numerical simulation of natural convection in a porous cavity filled with ferrofluid in presence of magnetic source.

[20] Kilic, M. (2018). Numerical investigation of heat transfer from a porous plate with transpiration cooling. Journal of Thermal Engineering, 4(1), 1632-1647.

[21] Pal, D., Mondal, H. (2013). Influence of thermophoresis and Soret-Dufour on magnetohydrodynamic heat and mass transfer over a non-isothermal wedge with thermal radiation and Ohmic dissipation. Journal of Magnetism and Magnetic Materials, 331, 250-255. 